\title{
HEART
}

\section{The potential for growth hormone in the management of heart failure}

The positive inotropic effect of growth hormone (GH) administration observed in normal subjects ${ }^{1}$ provides a rationale for the use of $\mathrm{GH}$ in cardiac failure and this is further supported by observations of the cardiac consequences of disturbed $\mathrm{GH}$ secretion. A relation between GH hypersecretion and cardiac morphology and function has been long recognised in the context of acromegaly, which is associated with a specific cardiomyopathy. ${ }^{2}$ Until recently the clinical effects of $G H$ deficiency were thought to be limited to reduced linear growth in children and possible impairment of glucose homoeostasis. It is now increasingly recognised, however, that $\mathrm{GH}$ deficiency in adults gives rise to a clinical syndrome characterised by abnormalities in body composition, with increased truncal adiposity, decreased lean body mass and bone density, reduced muscle power and exercise tolerance, and decreased general well being. ${ }^{3}$ In addition, retrospective epidemiological studies of hypopituitary subjects suggest premature cardiovascular mortality, ${ }^{4}$ which may be related to increased atherogenesis. ${ }^{5}$ Longstanding childhoodonset GH deficiency is associated with echocardiographic and radionuclide evidence of reduced cardiac output and diastolic dysfunction. ${ }^{67}$ Improvement in macrovascular disease with $\mathrm{GH}$ replacement seems possible but is so far unproven. However, the highly reproducible and beneficial effects of $\mathrm{GH}$ replacement therapy on cardiac function in the GH deficient adult raise the important theoretical possibility that GH therapy may have an additional role in the treatment of various syndromes of cardiac failure. In this editorial we highlight the established, important interactions between $\mathrm{GH}$ and cardiac structure and function and put into context the emerging evidence for beneficial effects of GH therapy in heart failure and in dilated cardiomyopathy in particular.

\section{Cardiac actions of $\mathbf{G H}$}

In most situations for which data are available, the peripheral actions of $\mathrm{GH}$ are dependent on the local production of insulin-like growth factor-I (IGF-I) which exerts local autocrine or paracrine effects. The evidence for direct and indirect effects of $\mathrm{GH}$ on cardiac function is based on the observation of cardiac GH receptor and IGF-I mRNA expression in animal models ${ }^{8}$ and an increment in cardiac IGF-I mRNA in response to $\mathrm{GH}$ administration. Furthermore, the development of ventricular hypertrophy in experimental models of hypertension in rats is associated with increased expression of IGF-I mRNA. ${ }^{10}$ In vitro studies of the direct effects of IGF-I on cardiac myocytes demonstrate enhancement of contractility ${ }^{11}$ and increased size of cultured cardiac myocytes. ${ }^{12}$ These findings are consistent with an important role for the GH/IGF-I axis in determining cardiac morphology and function although other factors which may be modified by GH specifically, including proto-oncogene expression and membrane $G$ protein/effector interactions, may play an as yet undefined part. The mechanism of IGF-I action on the heart is poorly defined but may include a combination of protein anabolic effects and, in animal models of long term GH excess, a paradoxical alteration in myosin heavy chain isoforms in favour of the V3 isoform; the latter is associated with a lower shortening velocity and ATPase activity and may therefore improve energy economy. ${ }^{13}$

\section{Effects of GH deficiency and excess on the heart} Clinical data suggest that growth hormone deficiency may exert a dual effect on the heart. The epidemiological studies linking adult onset hypopituitarism with premature cardiovascular mortality, ${ }^{4}$ although important, are limited by their retrospective nature and the fact that attribution of adverse effects to $\mathrm{GH}$ deficiency rather than other aspects of hypopituitarism may be invalid. Nonetheless, these studies have been paralleled by ultrasonic demonstration of intimal thickening and atherosclerotic changes in large arteries of hypopituitary patients. ${ }^{5}$ Furthermore hypopituitary patients have characteristic changes in body composition with increased central adiposity, hyperlipidaemia, relative insulin resistance, ${ }^{14}$ and increased circulating concentrations of fibrinogen and plasminogen activating inhibitor, all of which might theoretically predispose to atherogenesis. With the exception of insulin sensitivity, these abnormalities reverse during GH replacement therapy in the adult hypopituitary patient providing suggestive, indirect evidence of a causative association.

GH deficiency has also been shown to influence cardiac dimensions and function. This has been most clearly evident in childhood onset GH deficiency, which is characterised by a hypokinetic state with modest reductions in left ventricular (LV) wall mass, cardiac output, and blood pressure.$^{6}$ Data from studies in adult onset GH deficiency are variable with most documenting normal ventricular dimensions. ${ }^{15} 16$ The discrepancies may be due to heterogeneity in terms of blood pressure and duration of disease within the population with adult onset $\mathrm{GH}$ deficiency. Most studies of GH replacement therapy have demonstrated increased LV wall mass, stroke volume, and fractional shortening and improved diastolic function. ${ }^{6}{ }^{16}$ In addition, reduction in peripheral resistance during $\mathrm{GH}$ therapy has been reported, ${ }^{17}$ although this has not been confirmed by others. ${ }^{15}$ Most workers have also observed an improvement in exercise tolerance which has been attributed to improved cardiac function as well as to increased skeletal muscle mass and power. There is, as yet, no consensus on whether GH replacement therapy simply normalises cardiac dimensions or may go on to produce LV hypertrophy.

The effects of endogenous GH excess (acromegaly) on cardiac structure and function are of considerable interest in this context. ${ }^{2}$ In its earliest stages GH hypersecretion is 
associated with cardiac hyperkinesis which is manifest by increased myocardial contractility, enhanced cardiac output, and decreased peripheral resistance. Subsequently, myocardial hypertrophy occurs with impaired diastolic relaxation, probably owing to fibrotic change within the ventricle in addition to true ventricular hypertrophy. The final stage is one of ventricular dilatation with clinical evidence of cardiac decompensation, initially on effort and eventually with signs of congestive cardiac failure at rest. Because these changes evolve over several years, short term data on the cardiac effects of $\mathrm{GH}$ therapy must be interpreted with caution.

\section{GH therapy in heart failure}

Beneficial effects of $\mathrm{GH}$ have been documented in experimental animal models of heart failure. ${ }^{18}$ In addition in two cases there were reports of substantial benefits from the use of $\mathrm{GH}$ as adjunctive therapy for heart failure in humans. In the first of these, heart failure was due to a cardiomyopathy occurring in the context of insulin dependent diabetes, hypertension, and treated Cushing's disease with hypopituitarism; substantial haemodynamic improvement was evident within a few days of the start of pharmacological doses of GH (about six times that currently used for replacement doses in adults with GH deficiency). ${ }^{19}$ In the second there was an improvement in cardiac function, ventricular mass, and myofibrillar content of myocytes determined by electron microscopy in a patient with post partum hypopituitarism and dilated cardiomyopathy treated with GH. ${ }^{20}$ Further support for the use of GH in heart failure has now emerged from the findings of Fazio et $a^{21}$ who systematically studied the effects of GH in seven patients who had moderate to severe heart failure as a result of idiopathic dilated cardiomyopathy. The dose of GH utilised was four units on alternate days, giving a total dose similar to that currently used in the treament of $\mathrm{GH}$ deficient adults. Treatment for three months was associated with an increase in left ventricular wall thickness and reduction in chamber size (thereby reducing end systolic wall stress), improvement in cardiac output, both at rest and on exercise, and enhanced venticular work. Importantly, these improvements were achieved with a reduction in myocardial energy consumption and energy production, implying substantially improved energy efficiency. Furthermore, these beneficial changes were still partially evident when patients were restudied three months after treatment ended and most importantly, changes in subjective well-being paralleled the haemodynamic observations. Although the study was uncontrolled, the uniformity of beneficial effect in the study was striking and, given the underlying pathology, unlikely to be spurious. A possible confounding effect of GH-mediated enhanced conversion of thyroxine to triiodothyronine was not observed and $\mathrm{GH}$-induced antinatriuresis was not a clinical problem. However, GH therapy was associated with an increase in serum IGF-I into the range found in mild acromegaly which prompts obvious concerns that the short term benefits observed may be simply analogous to the temporary cardiac hyperkinesis of acromegaly which may progress to diastolic dysfunction and eventual cardiac decompensation.
How then should we use $\mathrm{GH}$ in the management of heart failure? The preliminary published data are certainly impressive. However, at present data on which to base treatment in end stage cardiac failure are limited although it is in this group that the risk/benefit ratio is likely to be most favourable. Much work remains to be done on devising $\mathrm{GH}$ dose schedules which achieve maximum haemodynamic benefit for the smallest increment in serum IGF-I and exploring the use of intermittent treatment schedules. Investigation of the effect of $\mathrm{GH}$ in causes of heart failure other than idiopathic dilated cardiomypathy is clearly indicated.

Department of Endocrinology,

St Bartholomew's Hospital,

London EC1A $7 B E$

1 Thuesen L, Christiansen JS, Sorensen KE, Jorgensen JOL, Orskov H, Henningsen $P$. Increased myocardial contractility following growth hormone administration in normal man. Dan Med Bull 1988;35:193-6.

2 Sacca L, Cittadini A, Fazio S. Growth hormone and the heart. Endocrine Revievos 1994;15:555-73.

3 De Boer H, Blok G-J, Van Der Veen EA. Clinical aspects of growth hormone deficiency in adults. Endocrine Reviews 1995;16:63-86.

4 Rosen T, Bengtsson B-A. Premature mortality due to cardiovascular disease in hypopituitarism. Lancet 1990;336:285-8.

5 Markussis V, Beshyah SA, Fisher C, Sharp P, Nicolaides AN, Johnston DG. Detection of premature atherosclerosis by high-resolution ultrasonography in symptom-free hypopituitary adults. Lancet 1992;340: 1188-92.

6 Amato G, Carella C, Fazio S, La Montagna G, Cittadini A, Sabatini D, et al. Body composition, bone metabolism, heart structure and function in growth hormone deficient adults before and after growth hormone replacement therapy at low doses. F Clin Endocrinol Metab 1993;77: 1671-6.

7 Cittadini A, Cuocolo A, Merola B, Fazio S, Sabatini D, Nicolai E, et al. Impaired cardiac performance in growth hormone deficient adults and its impaired cardiac performance in growth hormone deficient adults and its E219-E225.

8 Mathews LS, Enberg B, Norstedt G. Regulation of rat growth hormone receptor gene expression. $\mathcal{F}$ Biol Chem 1989;17:9905-10.

9 Isgaard J, Nilsson A, Vikman K, Isaksson OGP. Growth hormone regulates the level of insulin-like growth factor-I mRNA in rat skeletal muscle. $\mathcal{F}$ Endocrinol 1989;120:107-12.

10 Wahlander H, Isgaard J, Jennische E, Friberg P. Left ventricular insulinlike growth I increases in early renal hypertension. Hypertension 1992;19: 25-32.

11 Vetter U, Kupferscmid C, Lang D, Pentz S. Insulin-like growth factors and insulin increase the contractility of neonatal rat cardiocytes in vitro. Bas Res Cardiol 1988;83:647-54.

12 Ito $\mathrm{H}$, Hiroe M, Hirata $\mathrm{Y}$, Motoyoshi T, Adachi S, Masayoshi S, et al. Insulin like growth factor-I induces hypertrophy with enhanced expression of muscle specific genes in cultured rat cardiomyocytes. Circulation 1993;87:1715-21.

13 Timsit J, Riou B, Bertharat J, Wisnewsky C, Kato NS, Weisberg AS, et al. Effects of chronic growth hormone hypersecretion on intrinsic contractility, energetics, isomyosin pattern and myosin adenosine triphosphatase activity of rat left ventricle. $\mathcal{F}$ Clin Invest 1990;86:507-15.

14 Weaver JU, Monson JP, Noonan K, John WG, Edwards A, Evans KA, Cunningham $\mathrm{J}$. The effect of low dose recombinant human growth homone replacement on regional fat distribution, insulin sensitivity and cardiovascular risk factors in hypopituitary adults. $₹$ Clin Endocrinol Metab 1995;80:153-9.

15 Shahi M, Beshyah SA, Hackett D, Sharp PS, Johnston DG, Foale RA. Myocardial dysfunction in treated adult hypopituitarism: a possible explanation for increased cardiovascular mortality. Br Heart $\mathcal{f}$ 1992;67: 92-6.

16 Fort S, Weaver JU, Monson JP, Mills P. The effects of low-dose recombinant human growth hormone on cardiovascular structure and function in hypopituitary growth hormone deficient adults. Endocrinology and Metabolism 1995;2:119-26.

17 Caidahl K, Eden S, Bengtsson B-A. Cardiovascular and renal effects of growth hormone. Clin Endocrinol 1994;40:393-400.

18 Yang R, Bunting S, Gillett N, Clark R, Jin H. Growth homone improves cardiac performance in experimental heart failure. Circulation 1995;92: 262-7.

19 Cuneo RC, Wilmshurst P, Lowy C, McGauley G, Sonksen PH. Cardiac failure responding to growth hormone. Lancet 1989;1:838-9.

20 Frustaci A, Perrone GA, Gentiloni N, Russo MA. Reversible dilated cardiomyopathy due to growth hormone deficiency. Am $₹$ Clin Path 1992; 97:503-11.

21 Fazio S, Sabatini D, Capaldo B, Vigorito C, Giordano A, Guida R, et al. A preliminary study of growth hormone in the treatment of dilated cardiomyopathy. $N$ Engl $\Im$ Med 1996;334:809-14. 